\title{
Traffic Simulation Performance Optimization through Multi-Resolution Modeling of Road Segments
}

\author{
Daniel Zehe, David Grotzky, Heiko Aydt \\ TUM CREATE \\ 1 CREATE Way \\ 138602 Singapore \\ $+6566014015$ \\ \{daniel.zehe,david.grotzky,heiko.aydt\}@tum-create.edu.sg \\ Wentong Cai \\ Nanyang Technological University \\ 639798 Singapore \\ $+657904600$ \\ aswtcai@ntu.edu.sg \\ Alois Knoll \\ Technische Universität München (TUM) \\ 85748 Munich Germany \\ +498928918104 \\ knoll@in.tum.de
}

\begin{abstract}
In an agent-based traffic simulation the level of detail is crucial to the system's runtime performance as well as the fidelity of the results. Therefore, different model abstractions have been used throughout literature. Macroscopic, mesoscopic and microscopic models have their use-cases and benefits. Microscopic traffic simulations have a high level of detail but at the same time require a large amount of computational resources. In a large traffic network of a mega-city or an entire country, the use of a complete microscopic simulation is just not feasible. The resource required to do so are for most use-cases in no relation to the actual outcome. We propose a hybrid traffic simulation model that uses both, a high-resolution agent-based microscopic simulation alongside a lower resolution flow-based macroscopic simulation for specific road segments. The problem with using different simulation models is the fidelity at the boundary between such simulation models. This fidelity discrepancy is caused by the difficulties with aggregation and disaggregation passing through the boundary. We show, in this paper, that the computational performance (simulation time) can be improved by $20 \%$ while maintaining a relative high accuracy of below $5 \%$ deviation from a pure microscopic simulation.
\end{abstract}

\section{Categories and Subject Descriptors}

I.6.8 [Simulation and Modeling]: Types of SimulationDiscrete event; I.6.3 [Simulation and Modeling]: Applications; I.6.5 [Simulation and Modeling]: Model Development-Modeling methodologies

Permission to make digital or hard copies of part or all of this work for personal or classroom use is granted without fee provided that copies are not made or distributed for profit or commercial advantage, and that copies bear this notice and the full citation on the first page. Copyrights for third-party components of this work must be honored. For all other uses, contact the owner/author(s). Copyright is held by the author/owner(s).

SIGSIM-PADS'15, June 10-12, 2015, London, United Kingdom.

ACM 978-1-4503-3557-7/15/06.

http://dx.doi.org/10.1145/2769458.2769475.

\section{General Terms}

Performance

\section{Keywords}

Multi-Resolution Modeling; Macroscopic Modeling; Microscopic Modeling; Traffic Simulation; Aggregation; Disaggregation

\section{INTRODUCTION}

When simulating traffic, a fine grained view of the given problem is beneficial in many cases; especially when assessing the fragile interactions between driving behavior and the consequences on the vehicle models. For large traffic simulations with several (hundred-) thousand agents the processing power required is rather large. High performance computing is a viable option to decrease the computing time of a given simulation; however, access to such systems is not available to everyone.

There might be regions of a road network of an entire city or country where a lower level of detail is sufficient and computing resources can be saved. In such cases, a multiresolution model may offer a means to improve processing efficiency while only marginally affecting the overall simulation's fidelity. Such regions could be a highway between different on and off-ramps or long country roads connecting cities or villages.

The purpose of this paper is to present a prototypical design of a multi-resolution agent-based traffic simulation and evaluate its performance with regards to computational performance (decrease in execution time) and fidelity (deviation from the highest resolution). We present model switching between microscopically and macroscopically modeled road segments. We asses the performance gains and fidelity losses of the multi-resolution models at different microscopic to macroscopic ratios for a stretch of road between two intersections. 


\section{RELATED WORK}

There have been several approaches to multi-resolution modeling of traffic. Two main approaches towards the topic have been (1) the use aggregation and disaggregation at runtime and (2) the use multi-resolution entities.

The first approach, which aggregates a high resolution model at runtime to a lower resolution and then disaggregates from the low resolution to a high resolutio, is trivial on the aggregation side. This is because there is usually sufficient data to average over the existing high resolution agents' states. The disaggregation algorithm on the other hand has to interpolate or create/reconstruct information from the low resolution model in order to obtain the state information for the high resolution model. It has been shown that a frequent transition between high and low resolution models through aggregation and disaggregation is not good practice [12]. One problem is the thrashing effect, where agents cross between resolution boundaries often, can create a large overhead in computation; especially when running a distributed simulation.

This method of aggregation and disaggregation was initially developed for military simulations $[13,1]$, since it conforms very well with the command security structure in the military. Battalions, for example, are disaggregated divisions and allow manageable view on certain battlefield scenarios for different key personnel in the chain of command, whereas a disaggregated view of individual fighters is necessary for group leaders.

An approach to multi-resolution traffic modeling was discussed by Burghout [3, 2], where a ghosting method was used to ease the transition between microscopic and macroscopic boundaries in the aggregation based approach.

In order to avoid the trashing effects of frequent model switching, Natrajan et al. [11] propose the concept of MultiResolution entities (MRE). A MRE consolidates the properties of several resolutions in one object. Those properties are then kept consistent by design. This requires a larger memory footprint and individual operations require mode computing cycles for each of the resolutions, but there is no consistency problem.

Another approach to mitigate the trashing effects, especially for traffic simulations, while avoiding the use of MRE has been presented by Chua and Low [5], proposing a set of predictive algorithms. These algorithms are used to project an agent's future position on a road segment.

The multi-fidelity modeling approach presented by Choi et al. [4] describes generally how to convert an existing model into a multi-resolution model. This methodology can be used to increase the simulation speed for a given simulation as well as give measures for fidelity derivation. While their approach generalizes a methodology, this paper focuses on using multi-resolution modeling to investigate its potential in regard to a use in high performance traffic simulations.

\section{MODEL DESCRIPTION}

The multi-resolution model is comprised of a microscopic car-following model with lane changes and a macroscopic flow model. These models are executed simultaneously and are connected through a model-switching strategy to form a multi-resolution model of traffic on road segments. This section will begin with a detailed description of the two model types independently, followed by a description of the con- necting boundaries between two joining road segment zones. Such connections require aggregation agent states at the microscopic-macroscopic boundary and a disaggregation at the macroscopic-microscopic boundary.

The model uses a discrete time model for advancing the time in the microscopic and also macroscopic model at the same rate with fixed time intervals. The multi-resolution model has to function as a drop-in replacement for either macroscopic or microscopic simulations. This requires the two different models to have no knowledge of the respective other model and be fully self-sufficient traffic simulations.

\subsection{Microscopic Model}

For the microscopic traffic simulation models, a car-following model that determines acceleration and gap calculation between agents is important to express the traffic behavior correctly. The Intelligent Driver Model (IDM) developed by Triber et. al. [14] is widely used in agent-based traffic simulations. Together with the Minimal Overall Breaking Induced by Lane Change (MOBIL) [9] algorithms that extend the capabilities of IDM, this model is sufficient to model the driving behavior on a road segment. The acceleration for each agent is given by Equation 1;

$$
a_{\text {cur }}=a \cdot\left(1-\left(\frac{v_{\alpha}}{v_{0}^{\alpha}}\right)^{\sigma}-\left(\frac{s^{*}\left(v_{\alpha}, \Delta v_{\alpha}\right)}{s_{\alpha}}\right)^{2}\right)
$$

it uses the maximum acceleration of the agent $(\alpha)$ along with the current velocity of the agent as well as the position, derived using Equation 2

$$
s^{*}\left(v_{\alpha}, \Delta v_{\alpha}\right)=s_{0}+v_{\alpha} \cdot T+\frac{v_{\alpha} \cdot \Delta v_{\alpha}}{2 \sqrt{a \cdot b}}
$$

as well as $s_{\alpha}=x_{\alpha-1}-x_{\alpha}-l_{\alpha-1}$, and the velocity of the leading agent. Where $s_{0}$ denotes the minimum distance between two agents on one lane, $T$ is the time headway in seconds between agents in normal traffic conditions, and $a$ and $b$ are the comfortable acceleration and deceleration of the agent.

In addition to the IDM and MOBIL features, the underlying microscopic model is augmented to support arbitrary obstacles. Intersections, standing and moving vehicles are considered obstacles. An agent has to assess whether to adjust the acceleration or to change lanes in order to avoid collision with the obstacle. Intersections are a special type of obstacle that span the entire width of the road and have a transparency state. If the state is set to transparent, agents can pass through and normal car following models apply. Otherwise, agents will adjust their speed to come to a full stop. This allows for manipulation of the traffic simulation without violating any IDM or MOBILE rules.

\subsection{Macroscopic Model}

The macroscopic metrics described by Hoogendoorn and Bovy [7] are one way of expressing a macroscopic view on a road segment. They assume the traffic density $k$ can be derived, as in fluid dynamics, from the number of vehicles that pass through a certain section of road $(v c)$ in a given time and the length $(l)$ of that road segment (Eq. 3).

$$
k=\frac{v c}{l}
$$

This density, together with the average velocity $(\bar{v})$ of all agents within the given time frame, produces the traffic flow 
$q$ (Eq. 4)

$$
q=\bar{v} \cdot k
$$

Since the macroscopic model is not aware of individual agents within the segment, the model uses detectors at the boundaries to gain knowledge about entering and leaving agents. A front detector senses a microscopic agent entering the macroscopic zone and saves its ID as well as the cycle number (simulation time). The information gathered by the front-detector is used to calculate the vehicle's velocity at the end of the macroscopic zone and when a single vehicle should be passed to a connecting model. This model can either be a macroscopic, microscopic or even a multiresolution model. The front and back-detectors are illustrated in Figure 1. The three important metrics for macro-

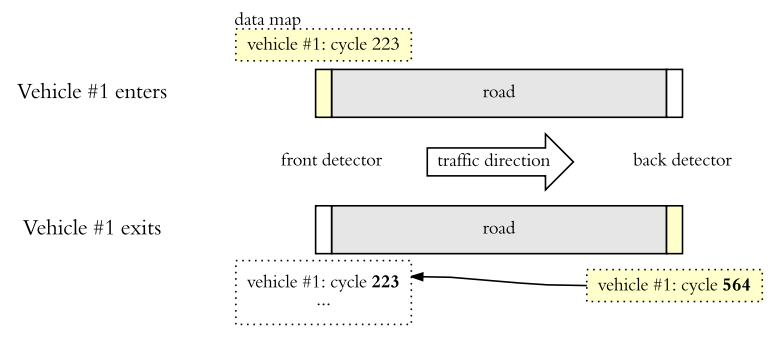

Figure 1: Using detectors to record entering and exiting vehicles

scopic traffic state are average velocity $(\bar{v})$, density $(k)$ and traffic flow $(q)$. The average velocity $(\bar{v})$ can be determined solely by the information gathered from the traffic detectors.

$$
\bar{v}=\frac{v c \cdot \bar{v}_{\text {old }}+v}{v c+1}
$$

An update to $\bar{v}$ is done whenever the back detector records a vehicle leaving the macro zone. This will implicitly decrease the vehicle count $(v c)$ by one, since the vehicle is not present in the data structure map assigned to this macroscopic zone. Because the number of vehicles on the road segment has changed, $k$ and $q$ values (Eq. 3 and 4 ) have to be updated as well.

\subsection{Multi-Resolution Model Extensions}

The microscopic and macroscopic simulations can be run independently in separate simulations. In a multi-resolution simulation they are executed simultaneously and have to exchange data. The points on a road segment at which data has to be exchanged are the front and back detectors discussed in Section 3.2. In our model we use two approaches for aggregation and disaggregation at the boundaries of the different zones. On the boundary between the microscopic and macroscopic zone (front detector), a position-triggered aggregation is used. The back-detector's boundary between the macroscopic and microscopic zone uses a cycle-based disaggregation scheme.

There are three different categories in which overall traffic can be categorized, they are dependent on the current traffic density $(k)$. Since they can be observed and/or calculated in macroscopic as well as microscopic zones, they are a good indicator for comparing macroscopic and microscopic road segments.
- Free Flow Traffic $k \leq k_{c}$ : The vehicles on the road segment are able to achieve their desired velocity by accelerating normally. This critical density $\left(k_{c}\right)$ has been described by Hoogendoorn and Bovy [7] and is the density at which congestion starts.

- Congested Traffic $k_{c} \leq k \leq k_{j}$ : Since traffic density that exceeds $k_{c}$ leads to congestions, vehicles can not accelerate as freely as before and might not reach their desired velocity. The vehicles also have not yet come to a full stop that $k$ would be reaching the jam density $k_{j}$. Intermittent breaking of agents can be observed. This traffic state has been described by Helbing et. al [6].

- Jammed Traffic $k=k_{j}$ : When the density of a road segment reaches $k_{j}$ as described by Hoogendoorn and Knoop [8]. All vehicles come to a full stop and no movement is possible.

\subsubsection{Aggregation}

In the microscopic area before the aggregation boundary (micro-zone) the simulation checks during each simulation cycle what agents traverse though the defined position where the micro-zone ends and the macro-zone starts. For estimating the projected cycle time for disaggregating the vehicle back into the micro-zone, two operation modes are possible. One is the single-lane mode where overtaking is not possible and the other one is the multi-lane mode.

In the single-lane case, the vehicle's velocity $(v)$ before passing into the macro-zone is taken, together with the length of the macro-zone $\left(l_{\text {macro }}\right)$, to determine the number of simulation cycles that have to pass before the vehicle is disaggregated back into the micro-zone at the back detector.

$$
\Delta \text { cycles }=\frac{l_{\text {macro }}}{v \cdot t_{\text {cycle }}}
$$

When looking at the model for a multi-lane road segment, the aggregation and cycle prediction gets more complicated. Since overtaking is possible, a vehicle's maximum velocity is not restricted by the leading vehicle on the same lane but also by the vehicle furthest away on any of the parallel lanes. Therefore, the projection takes the traffic density $(k)$ immediately after the macro-zone into account as well. The three cases of traffic state as discussed above have to be regarded.

For the free flow traffic case, the acceleration $(a)$ is regarded for the entire length of the macro-zone. The total travel time through the macro-zone is determined using Equations 7, 8 and 9 .

$$
\begin{gathered}
l=\frac{a}{2} \Delta t^{2}+v \cdot \Delta t \\
\Delta t=\frac{-v+\sqrt{v^{2}+2 \cdot a \cdot l}}{a} \\
\Delta \text { cycles }=\frac{\Delta t}{t_{c y c l e}}
\end{gathered}
$$

Should the velocity at the end of the macro-zone $\left(v_{\text {end }}=\right.$ $a \Delta t+v)$ exceed the desired velocity $v_{0}$, the total travel time has to be recalculated by having an acceleration time $\left(\Delta t_{a}\right)$ and a constant velocity phase $\left(\Delta t_{v 0}\right)$.

$$
\Delta t_{a}=\Delta t-\frac{v_{e n d}-v_{0}}{a}
$$




$$
l_{a}=\frac{a\left(\Delta t_{a}\right)^{2}}{2}+v \Delta t_{a}
$$

The constant velocity phase is calculated using Equation 12.

$$
\Delta t_{v 0}=\frac{l-l_{a}}{v_{0}}
$$

Where $t_{v 0}$ is the time spend in constant velocity $v_{0} . l$ and $l_{a}$ represent the total distance of the macro-zone and the distance traveled while accelerating respectively.

The cycle count when the vehicle exits the macro-zone is then determined by Equation 13.

$$
\Delta \text { cycles }=\frac{\Delta t_{a}+\Delta t_{v 0}}{t_{c y c l e}}
$$

For the congested traffic state, the acceleration is determined as in the free flow case (Eq. 7,8,9), but then multiplied with a density proportion factor $p_{\text {dens }}$ which leads to an adjusted acceleration value $a^{\prime}$. This is necessary to cope for very slow moving traffic and to counteract unrealistic acceleration and breaking behaviors. The density proportion factor is calculated using Equation 14 with the densities from above.

$$
p_{\text {dens }}=\frac{k-k_{c}}{k_{j}-k_{c}}
$$

The cycle count is then determined as it would in the free flow case, except the acceleration is adjusted.

In the traffic jam case, the velocity at the back detector boundary is 0 and the projection is performed using the vehicle's velocity and zero acceleration.

Since both, the single and multi-lane calculation of $\Delta$ cycles, calculate the time a vehicle spends in the macroscopic simulation, the returned position $\left(x_{r e t}=x+l_{\text {macro }}\right)$ is equal with the back detector's position. The absolute cycle in which the vehicle is put back into the microscopic simulation, after removing it, is determined using Equation 15.

$$
\text { cycle }_{\text {ret }}=\text { cycle }_{\text {now }}+\Delta \text { cycles }
$$

IDM car-following model relies very much on a leading vehicle to determine the new acceleration. When there is no vehicle leading, a free-flow mode is chosen. The freeflow mode in the IDM model is much simpler and assumes the maximum acceleration. However, once an agent is removed from the microscopic model, a following vehicle loses its leading vehicle to base its acceleration algorithms on (Eq 1). Free-Flow traffic mode is not applicable to the following vehicle, since it could lead to unwanted acceleration. Therefore, we introduce ghost vehicles to mitigate this problem [3]. A ghost vehicle contains only a subset of a true microscopic agent and is used to approximate a microscopic model. A ghost vehicle is spawned as soon as

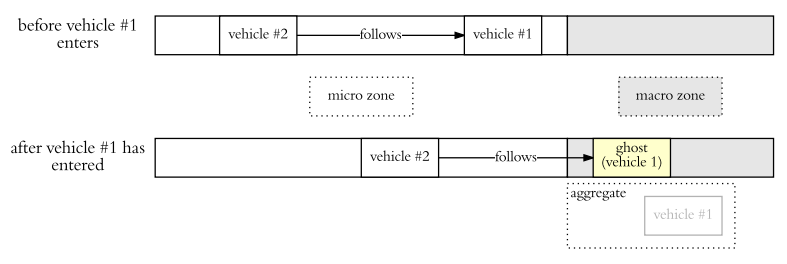

Figure 2: Ghost vehicle generation at micro-macro boundary

a vehicle traverses through the micro-macro boundary. At this point the ghost's length, position and velocity are set to the properties of the removed agent's. Each cycle, the ghost's microscopic model states are updated by the multiresolution component. This tricks the subsequent agent into following into the macro-zone. A ghost moves along the macro-zone until another vehicle enters the macro-zone. At that point the ghost is discarded and replaced by a new one. The movement of a ghost is dependent on the number of lanes (single or multi-lane mode) and the traffic state behind the macro-zone. In single lane mode, the ghost moves along the macro-zone at a constant velocity it had when it was created. In a multi-lane scenario, the traffic state in the microscopic zone behind the macro-zone is used to modify the ghost's velocity. The new velocity $v^{\prime}=(1-$ visc $) \cdot v$ uses a viscosity metric that is derived from the traffic density behind the macro-zone and given by Equation 16 (similar to the density proportion factor $p_{\text {dense }}$ ).

$$
\operatorname{visc}= \begin{cases}\frac{k-k_{c}}{k_{j}-k_{c}} & k_{c} \leq k \leq k_{j} \\ 1 & k>k_{j}\end{cases}
$$

At each cycle the density inside the macro zone is queried and if it exceeds the jam density $k_{j}$ the ghost is stopped. This leads to agents reducing their speed when entering the macro-zone and eventually stopping agents before entering the macro-zone. Since the macro density is updated whenever a vehicle is disaggregated back into the microscopic model, movement of the ghost continues when $k_{\text {macro }}$ falls below $k_{j}$. Should a vehicle reach the end of the macro-zone before being replaced, it is discarded.

\subsubsection{Disaggregation}

When vehicles are returned to the macroscopic-microscopic boundary disaggregation, has to be performed. There are 3 steps involved to ensure the model integrity is not violated.

1. Depending on the current cycle time, the internal data structure holding all agents currently in the macrozone is checked, and all agents that have a lower return cycle number $\left(\right.$ cycle $\left._{r e t}\right)$ are prepared to be returned to the microscopic zone. The data structure is depicted in Figure 3

2. If there is a vehicle that is supposed to be returned at the current cycle, the lane(s) are checked for enough free space to insert the vehicle safely.

3. Once a vehicle is supposed to be returned at a specific location, the IDM calculation is executed. It is then checked if an emergency breaking situation would occur. If this is the case, insertion is suspended. This check is not executed when the average velocity of the micro-zone is below $10 \%$ of the roads recommended speed, in order to allow for insertion when the traffic state is strongly congested.

For a multi-lane scenario, all lanes are checked against the free-space and emergency breaking conditions. Should the checks fail for every single lane, the disaggregation is postponed. Vehicles stay in the data structure and are checked again on the next cycle. On a successful disaggregation the vehicles are removed from the data structure and the backdetector is notified. 


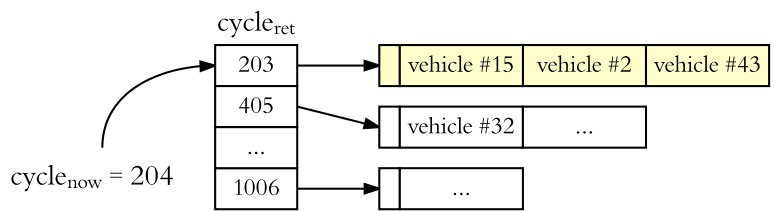

Figure 3: Microcscopic vehicle date holding data structure

\section{MODEL EVALUATION}

Since the aim of the proposed multi-resolution model is tc increase the performance while affecting the model fidelity as little as possible, we compare the multi-resolution performance against a pure microscopic simulation.

\subsection{Design of Experiments}

We design three experiments with the following setup in order to establish a microscopic base line for comparison and experiments for evaluating performance and fidelity respectively.

- The Microscopic Mode simulates the entire road segment using only the microscopic model. There is no need for model switching and it serves as the base-line implementation we are going to compare the performance and fidelity against (compare Figure 4a).

- The Multi-resolution Mode simulates a road segment as discussed in Section 3. Both microscopic and macroscopic models are used and model-switching at the boundaries is executed (compare Figure 4b). This is done to gain the performance measures that are later compared to the base-line microscopic model experiment.

- The Dual-Simulation Mode runs the previous modes simultaneously with identical starting conditions. The mode is used to evaluate the fidelity effects of multiresolution execution (compare Figure 4c).

a

microscopic mode
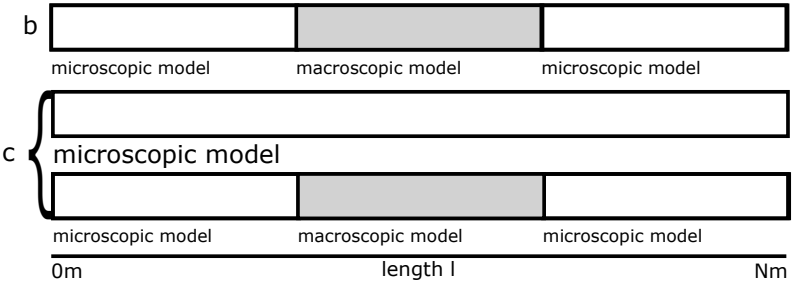

Figure 4: Simulation experiment setups

We have chosen 4 different driver-vehicle unit configurations for the microscopic road segments. They are normal, timid and aggressive driver as well as trucks. Each of the configurations have different values for the preferred maximum speed, the time headway, the acceleration and deceleration for normal traffic conditions. These values for different types have been identified by Kesting et al. [10]. In order

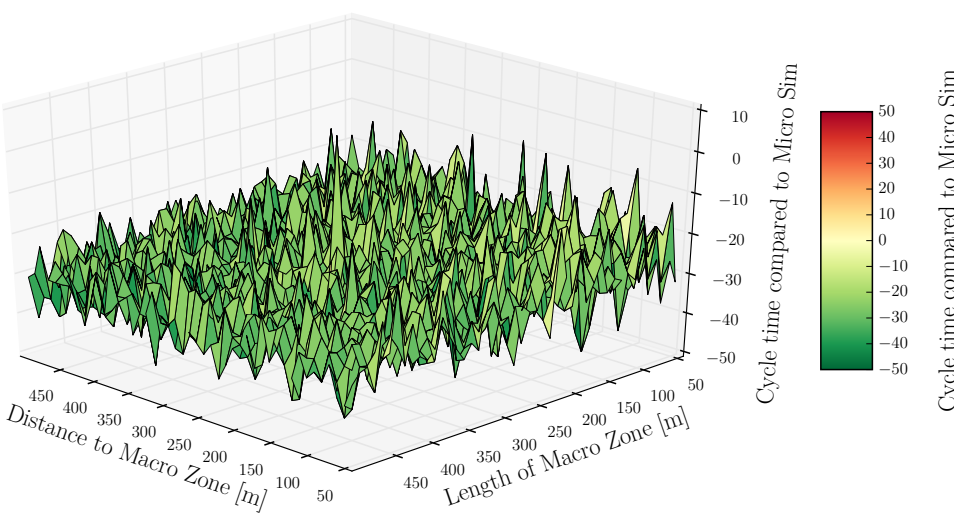

Figure 5: Single lane performance comparison: Relative performance between microscopic and multiresolution mode. Cycle time up to $40 \%$ reduced for long macro-zones and long distance to macro-zone.

to generate a heterogeneous agent population, the properties are uniformly distributed around the values identified by Kestig et al. with a margin of $\pm 20 \%$.

\subsection{Model Performance}

The goal of this paper is to evaluate the performance increase when using multi-resolution models for traffic simulations. Therefore, we compare the average processing of one processing cycle in the simulation for a pure microscopic simulation $\left(\bar{t}_{m i c r o}\right)$ and a multi-resolution simulation $\left(\bar{t}_{m r s}\right)$. The performance difference $(\Delta p)$ was then calculated using Equation 17.

$$
\Delta p=\frac{\bar{t}_{\text {mrs }}}{\bar{t}_{\text {micro }}}-1
$$

A close-to-zero The experiment run several $(>20)$ simulations in microscopic mode (Figure 4 a) as well as multiresolution mode (Figure $4 \mathrm{~b}$ ). Each simulation created 15 agents and stopped them at the same location within each simulation with a non-transparent obstacle. Once all agents fully stop, the obstacle are made transparent. This allows the IDM to accelerate the agents and move along the road segment. For each simulation cycle the exection time is recorded and then averaged over the entire simulation until all agents reach the end of the road segment.

value of $\Delta p$ indicates that the performance of both simulations is equal, whereas negative values indicates less processing time is spend on a cycle in the multi-resolution simulation. The performances for single- and multi-lane simulations are shown in Figues 5 and 6 . It shows that for the single-lane mode the performance increases when the macrozone becomes longer. An increase in model performance is also seen, even though not so prominently visible, when the distance to the macro-zone gets longer. The same general shape can be seen in the multi-lane mode. There, the overall performance increase is, averaged over all configurations, bigger $(+30 \%$ compared to $+25 \%)$. Nevertheless, the individual data-points can show a higher performance increase. It also shows that there is a larger deviation from the mean value. The performance increase is bigger due to the more complex model in the pure microscopic simulation. There is of course an overhead due to the model switching mechanism. The mechanism includes the aggregation of the agent 


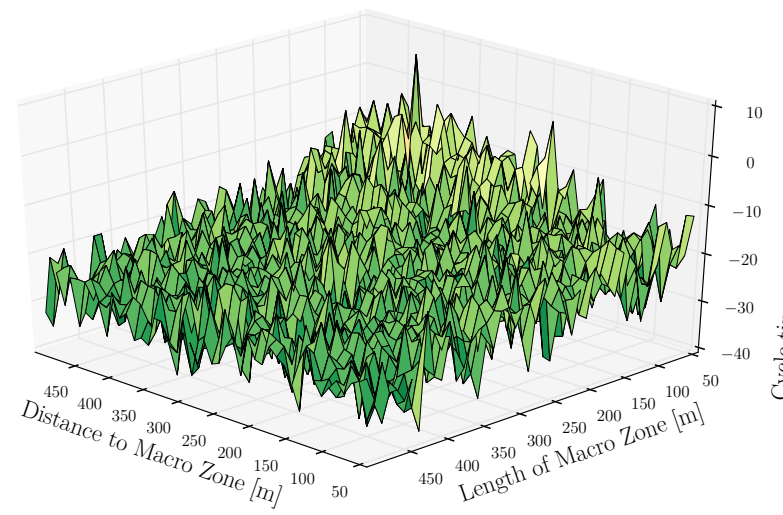

Figure 6: Dual lane performance comparison: Relative performance between microscopic and multiresolution mode. Cycle time reduction lessens when decreasing the length of the macro-zone.

at the front-detector, where the agent is removed from the microscopic simulation, the estimated re-entry cycle is calculated and a ghost vehicle is created; and the disaggregation, where the agents is put back into the microscopic simulation (see Sections 3.3.1 and 3.3.2). This overhead with regard to the entires simulation run is shown in Table 1.

\begin{tabular}{lcccc} 
& $\min \%$ & $\max \%$ & $\operatorname{mean} \%$ & $\sigma$ \\
\hline single-lane & 9.3 & 14.5 & 9.4 & \pm 0.39 \\
multi-lane & 6.5 & 10.8 & 7.6 & \pm 0.74
\end{tabular}

Table 1: Model switching overhead

\subsection{Model Fidelity}

The model fidelity is determined between the multi-resolution simulation and the pure-microscopic simulation using the experimental setup Dual-Simulation Mode. In order to quantify the differences we have to execute both simulations with the exact same starting parameters and settings. This leads to each agent in the microscopic simulation having an exact counterpart in the multi-resolution simulation. We are evaluating two types of deviations that occurs from the switching and the macroscopic model:

- Agent State Deviation describes the difference between the interval state variables.

- Differences in Traffic Flow is a more statistical assessment of the influence of the multi-resolution simulation. It is a macroscopic metric used instead of the individual state based agents fidelity.

\subsubsection{Agent State Deviation}

In order to determined the agent state deviation by the proposed multi-resolution model, we use the dual-simulation mode simulation with a road length of 5000 meters and 100 vehicles. Vehicles are spawned with a distribution of $3: 3: 3$ : 1 of the vehicles classes discussed previously. Both simulations contain a non-transparent obstacle at the exact same position and are only opened when all agents have come to a full stop. This obstacle will mimic an intersection, where a defined number of vehicles are release at the same time. This intersection is always placed before the macroscopic zone (front detector). In the multi-resolution similation model a macroscopic zone is placed at the 2000 ${ }_{24}^{32}$ meter mark. This is not present in the pure microscopic ${ }_{16}^{24}$ simulation. The dual simulation mode setup is illustrated in Eigure 7. Once a vehicles leaves the macroscopic zone, 苋

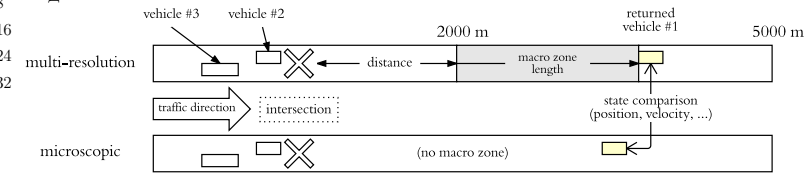

Figure 7: Dual-Simulation setup for agent stat deviation experiment

its state is logged in each of the simulations and analyzed after the simulation finishes. The root-mean-square for the position $\left(x_{r m s}\right)$ and velocity $\left(v_{r m s}\right)$ deviation are calculated.

$$
\begin{gathered}
y_{r m s} \in\left\{x_{r m s}, v_{r m s}\right\} \\
y_{r m s}=\sqrt{\frac{\sum_{i=1}^{N}\left(y_{m r s}^{i}-y_{m i c r o}^{i}\right)^{2}}{N}}
\end{gathered}
$$

Since the rms value grows with positive and negative value differences, it only gives absolute discrepancy information. A more fitting estimate for over- and underestimation of the agent state variables is the root mean square of the ratio $\left(y_{r m s}^{\%}\right)$ between multi-resolution simulation and pure microscopic simulation.

$$
y_{r m s}^{\%}=\sqrt{\frac{\sum_{i=1}^{N}\left(\frac{y_{m r s}^{i}}{y_{\text {micro }}^{i}}\right)^{2}}{N}}-1
$$

If the root-mean-square ratio is 0.0 then there is no significant difference between the microscopic and multi-resolution simulation, but if the value is negative, the multi-resolution vehicles have a smaller value (e.g. position behind microscopic simulation). This means they were underestimated when aggregating. Should the value be positive the multiresolution vehicles overestimated their projection values. These experiments were repeated 20 times and the standard deviation $(\sigma)$ to all values was calculated.

$$
\sigma_{y}=\sqrt{\frac{\sum_{i=1}^{20}\left(y_{r m s}^{i}-\bar{y}_{r m s}\right)}{20-1}}
$$

The experiments were conducted with different ratios of microscopic and macroscopic regions. This was done by changing the length of the macro-zones without changing the overall road segment length constant. This leads to an increasing proportion of the road segment being handled by the macroscopic model. We choose macro-zones length between 50 and 500 meters with 10 meter increments (45 configurations). Additionally, we varied the distance from the intersection to the beginning of the macro-zone. The same 50 to 500 meter and 10 meter increment was applied there (45 configurations). This lead to a total number of 40,500 simulation runs $(45 \cdot 45 \cdot 20)$ in single lane mode and the same amount in multi-lane mode.

The two parameters discussed previously are shown on the $\mathrm{x}$ an $\mathrm{y}$-axis of Figure 8 . The $\mathrm{z}$-axis shows the positional deviation in percent between the microscopic and 


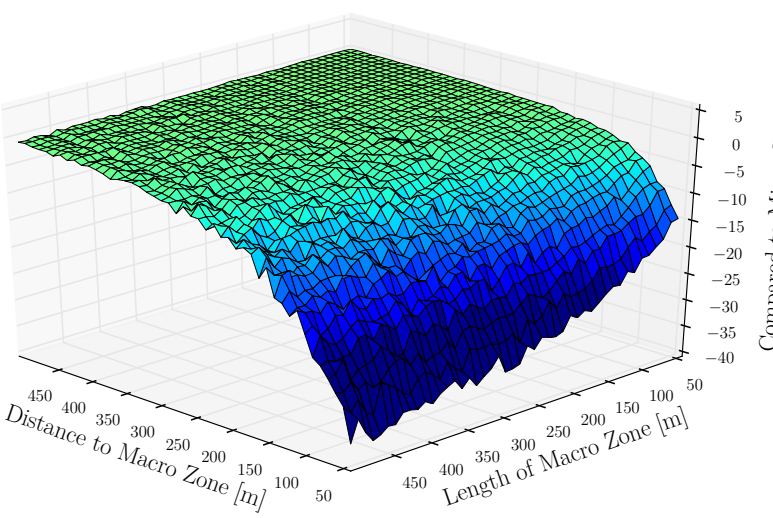

Figure 8: Position deviation in percent on singlelane mode: Longer macro-zones and short distances to the macro-zone show the highest positional difference. Small deviation visible for longer distance to the macro-zone regardless of the length of the macro-zone.

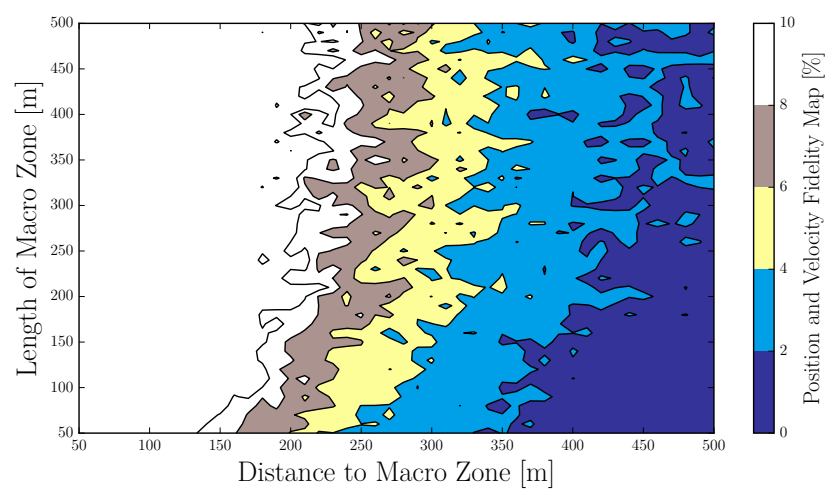

Figure 9: Position and velocity deviation map for single-lane mode: Maximum percent deviation for either velocity or position dependent on the distance to macro-zone and the length of the macrozone. Distance to macro-zone bigger influence than the length.

multi-resolution simulation. A negative value stands for an underestimation of the projected position after disaggregation.

In the single-lane scenario the distance to the macro-zone is more important than the length of the macro-zone itself. This can be observed since the graph falls down when the distance to the macro zone is below 200 meters for the distance to the macro-zone but not when the length is changed. This only has a significant effect when the distance to the macro-zone is already low.

For the multi-lane mode (number of lanes between 2 and 5) the results are similar. It also shows that the distance to the macro-zone has a smaller influence than the length. Short macro-zones and long distance to the macro-zone have the least influence on the deviation of position.

The map in Figure 9 shows the absolute maximum deviation from either position or velocity (worst case for deviation) for the single-lane mode. It can be seen that the distance to the macro-zone has a bigger influence and is more

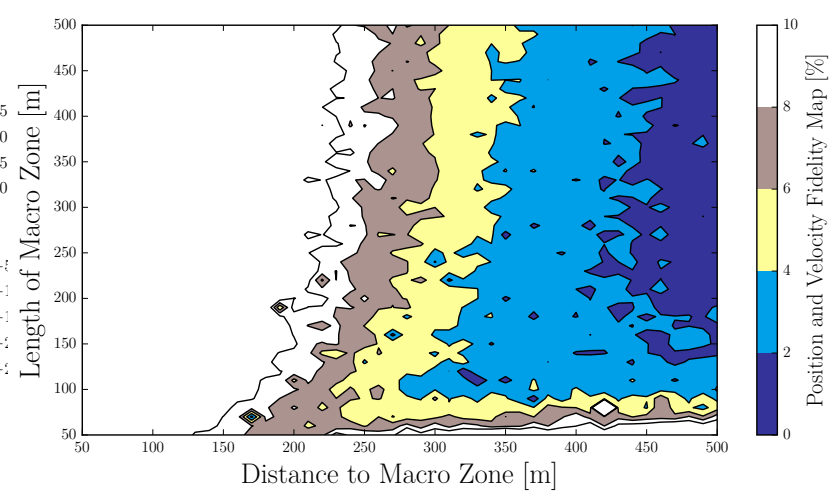

Figure 10: Position and velocity deviation map for multi-lane mode with three lanes: Maximum percent deviation for either velocity or position dependent on the distance to macro-zone and the length of the macro-zone. Distance to macro-zone has bigger influence than length, except for very short macrozones.

volatile. For road segments that are at least $200 \mathrm{~m}$ the deviation is below $10 \%$. The same is shown in Figure 10, except that there are fewer areas with less than $2 \%$ deviation and more configurations lead to a higher than $10 \%$ deviation. Also it can be seen that the distance to macro-zone is of influence since the fidelity gets worse when the length of the macro-zone is really short.

\subsubsection{Traffic Flow Deviation}

After looking at the agents' state deviation, the comparison of the traffic flow gives a more statistical approach to the influence of the multi-resolution simulation. Therefore, the region behind the macro-zone is examined. The tested scenario is depicted in Figure 11, where the observed area in both simulations is shown. We logged the traffic flow in both simulations from the cycle when the first vehicle enters the zone in either simulation until the simulation run ended. A student's-t test, to disprove the hypothesis

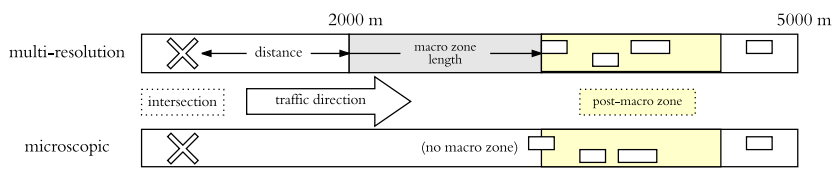

Figure 11: Post macro zone flow comparison

that there is no significant difference between the two zones, has been conducted. This test shows that for a single-lane $(p \leq 0.773)$ and a dual-lane $(p \leq 0.879)$ the null-hypothesis cannot be discarded and therefore the traffic flow in both simulation shows no significant difference by introducing the multi-resolution model.

\section{CONCLUSION}

The evaluation shows that a more than $20 \%$ performance increase compared to a pure microscopic model can be achieved. The main factors that influence the relative performance (pure microscopic vs. multi-resolution) is the complexity of 
the high resolution model and the length of the road segment. The longer the road segment, the more time that can be spent in the macro-zone and therefore less computing needs to be done. The side-effect of this is that the fidelity of the result decreases as well. It also shows that a lower than $6 \%$ fidelity deviation on single-lane roads can be kept. This is given the macroscopic zone is at least $150 \mathrm{~m}$ from an intersection. For a dual-lane road segment, a deviation of less then $6 \%$ can be achieved when the macro-zone is at least $300 \mathrm{~m}$ from an intersection and $200 \mathrm{~m}$ long. We have also shown that the introduction of a macroscopic element into a microscopic simulation does not change the traffic flow behind a macroscopic zone modeled.

In this paper we have shown that a simple multi-resolution approach to agent-based traffic simulations can have a great improvement in the performance of the simulation. Nevertheless, the performance improvement comes at a price of model fidelity. We have shown that with macroscopic metric there is no significant difference between a multi-resolution simulation and a pure-microscopic. With microscopic measures a loss in fidelity is observed; however, depending on the road configuration, this can be reduced to a minimum. For a city scale scenario we have statically analyzed the potential performance gain and fidelity loss.

Future research should look into the dynamic creation of multi-resolution road segments depending on the road conditions. Also, the static analysis for an entire country could lead to an even bigger performance increases, since connecting highways or country roads are longer and offer more macroscopic zones that are easier to predict.

\section{ACKNOWLEDGMENTS}

This work was financially supported by the Singapore National Research Foundation under its Campus for Research Excellence And Technological Enterprise (CREATE) programme.

\section{REFERENCES}

[1] M. Adelantado and P. Siron. Multiresolution Modeling and Simulation of an Air-Ground Combat Application. In proceedings of the 2001 Spring Simulation Interoperability Workshop, Orlando, USA, 25-30 Mar., 2001. IEEE.

[2] W. Burghout. Hybrid microscopic-mesoscopic traffic simulation. Doctoral dissertation, Royal Institute of Technology, Stockholm, 2004.

[3] W. Burghout and H. Koutsopoulos. Hybrid Traffic Simulation Models: Vehicle Loading at Meso-Micro Boundaries. In proceeding of the International Symposium of Transport Simulation, Lausanne, Switzerland, 04-06 Sep., 2006.
[4] S. H. Choi, S. J. Lee, and T. G. Kim. Multi-fidelity modeling \& simulation methodology for simulation speed up. In proceedings of SIGSIM-PADS '14, pages 139-150, New York, New York, USA, 18-21 May, 2014. ACM.

[5] B. Chua and M. Low. Predictive algorithms for aggregation and disaggregation in mixed mode simulation. In proceeding of the Winter Simulation Conference, pages 1356-1365, Austin, Texas, USA, 13-16 Dec., 2009. IEEE.

[6] D. Helbing and A. Hennecke. Micro-and macro-simulation of freeway traffic. Mathematical and computer modelling, 35(5):517-547, 2002.

[7] S. Hoogendoorn and P. Bovy. State-of-the-art of vehicular traffic flow modelling. Proceedings of the Institution of Mechanical Engineers, Part I: Journal of Systems and Control Engineering, 215(4):283-303, 2001.

[8] S. Hoogendoorn and V. Knoop. Traffic flow theory and modelling. In D. Banister, B. Van Wee, and J. A. Annema, editors, The Transport System and Transport Policy: An Introduction, pages 125-159. Edward Elgar Publishing Ltd, Cheltenham, United Kingdom, 2012.

[9] A. Kesting, M. Treiber, and D. Helbing. General Lane-Changing Model MOBIL for Car-Following Models. Transportation Research Record: Journal of the Transportation Research Board, 1999:86-94, 2007.

[10] A. Kesting, M. Treiber, and D. Helbing. Agents for traffic simulation. In A. M. Uhrmacher and D. Weyns, editors, Multi-Agent Systems: Simulation and Applications, pages 325-356. CRC Press, Boca Raton, Florida, USA, 2008.

[11] A. Natrajan. MRE: a flexible approach to multi-resolution modeling. In proceedings of the 11th Workshop on Parallel and Distributed Simulation, pages 156-163, Lockenhaus, Austria, 10-13 June, 1997. IEEE.

[12] P. F. ReynoldsJr, A. Natrajan, and S. Srinivasan. Consistency maintenance in multiresolution simulation. Transactions on Modeling and Computer Simulation (TOMACS), pages 368-392, 1997.

[13] G. Tan, W. Ng, and F. Moradi. Aggregation/disaggregation in HLA multi-resolution distributed simulation. In proceedings of the IEEE/ACM International Symposium on Distributed Simulation and Real Time Applications, pages 76-83, Cincinnati, Ohio, USA, 13-15 Aug., 2001. IEEE.

[14] M. Treiber, A. Hennecke, and D. Helbing. Congested traffic states in empirical observations and microscopic simulations. Physical Review E, 62:1805-1824, Aug. 2000 . 